\title{
WILD AUTOMORPHISMS OF PROJECTIVE VARIETIES, THE MAPS WHICH HAVE NO INVARIANT PROPER SUBSETS
}

\author{
KEIJI OGUISO AND DE-QI ZHANG
}

\begin{abstract}
Let $X$ be a projective variety and $\sigma$ a wild automorphism on $X$, i.e., whenever $\sigma(Z)=Z$ for a non-empty Zariski-closed subset $Z$ of $X$, we have $Z=X$. Then $X$ is conjectured to be an abelian variety with $\sigma$ of zero entropy (and proved to be so when $\operatorname{dim} X \leq 2)$ by Z. Reichstein, D. Rogalski and J. J. Zhang in their study of projectively simple rings. This conjecture has been generally open for more than a decade. In this note, we confirm this original conjecture when $\operatorname{dim} X \leq 3$ and $X$ is not a Calabi-Yau threefold, and also show that $\sigma$ is of zero entropy when $\operatorname{dim} X \leq 4$ and the Kodaira dimension $\kappa(X) \geq 0$.
\end{abstract}

\section{Contents}

1. Introduction 1

2. Preliminary results in arbitrary dimensions 3

3. Preliminary results in lower dimensions 10

4. Proof of Theorem 1.2 when $q=2 \quad 13$

5. Proof of Theorem 1.2 when $q=1 \quad 15$

6. Proof of Theorem 1.5 19

7. Wild automorphisms of zero entropy 22

References $\quad 24$

\section{INTRODUCTION}

We work over the complex number field $\mathbb{C}$, unless stated otherwise (cf. Proposition 2.1). Let $X$ be a projective variety. An automorphism $\sigma$ in $\operatorname{Aut}(X)$ is called wild in the sense of [21] if: whenever $\sigma(Z)=Z$ for a non-empty Zariski-closed subset $Z$ of $X$, we have $Z=X$, or equivalently, for every $x \in X$, the orbit $\left\{\sigma^{s}(x) \mid s \geq 0\right\}$ is Zariski dense in $X$. This always holds when $\operatorname{dim} X=0$.

2010 Mathematics Subject Classification. 14J50, 32M05, 11G10.

Key words and phrases. Wild automorphism, Abelian variety, Fixed point, Entropy.

The first named author is partially supported by JSPS and NCTS and the second named author is partially supported by an ARF of NUS. 
In this note, we will prove Theorems 1.2 and 1.5 below which support the following conjecture of Z. Reichstein, D. Rogalski and J. J. Zhang ([21, Conjecture 0.3]).

Conjecture 1.1. Assume that a projective variety $X$ admits a wild automorphism. Then $X$ is isomorphic to an abelian variety.

Once $X$ is known to be an abelian variety (as asserted in Conjecture 1.1), the wild automorphisms on $X$ can been completely classified as in [21, Theorem 0.2]; in particular, they have zero entropy. We recall that for a compact Kähler manifold (or smooth projective variety) $X$ of dimension $n \geq 1$, and $f \in \operatorname{Aut}(X)$, we let $d_{i}(f)$ be the $i$-th dynamical degree of $f$, that is, the spectral radius of $\left.f^{*}\right|_{H^{i, i}(X, \mathbb{R})}$. The well-known log concavity of dynamical degrees due to Khovanskii and Teissier asserts that

$$
\text { (*) } d_{i-1}(f) d_{i+1}(f) \leq d_{i}(f)^{2}
$$

for all $1 \leq i \leq n-1$. Hence $d_{i}(f)=1$ for one $i$ with $1 \leq i \leq n-1$ implies that it holds for all such $i$. The classical results of Gromov and Yomdin imply that the topological entropy

$$
h(f)=\log \max _{1 \leq i \leq n}\left\{d_{i}(f)\right\} .
$$

Hence $f$ has zero entropy if and only if $d_{1}(f)=1$.

When $\operatorname{dim} X \leq 2$, Theorem 1.2 is proved in [21, Theorem 6.5] (or Theorem 3.1 for a slightly simplified proof there). See [10], and also our Section 6 for results when $X$ has Kodaira dimension zero. Theorem 1.2 follows from Propositions 3.2, 4.1, 5.2 and 6.4.

Theorem 1.2. Let $X$ be a projective variety over $\mathbb{C}$ of dimension $\leq 3$. Assume that $X$ admits a wild automorphism $\sigma$. Then either $X$ is an abelian variety, or $X$ is a Calabi Yau manifold of dimension three and $\sigma$ has zero entropy.

Here, a projective variety $X$ is a Calabi Yau manifold if its canonical line bundle $\mathcal{O}\left(K_{X}\right)$ is torsion and its topological fundamental group is finite.

Remark 1.3. We believe that a Calabi-Yau threefold $X$ should not have any wild automorphism $\sigma$. This is confirmed if [18, Question 2.6] (= Conjecture 7.3) is affirmative for $X$ and this is also equivalent to the projective simplicity of the twisted homogeneous coordinate (non-commutative) ring of any $\sigma$-ample line bundle of $\sigma$ with zero entropy (cf. [21, Proposition 2.2] and [9, Theorem $1.2(2)]$ ). See Section 7 for details.

Let $X$ be a projective variety. As is already indicated in Remark 1.3, the motivation of studying wild automorphism arises from [21, Proposition 0.1] where it is proved that the twisted homogeneous coordinate ring

$$
B(X, L, \sigma)=\oplus_{n=0}^{\infty} H^{0}\left(X, \otimes_{i=0}^{n-1}\left(\sigma^{i}\right)^{*} L\right)
$$


associated to any $\sigma$-ample line bundle $L$ on $X$ is projectively simple if and only if $\sigma$ is a wild automorphism on $X$ (cf. [21, Proposition 2.2]); every ample line bundle on an abelian variety is $\sigma$-ample if $\sigma$ is wild ([21, Corollary 8.6]). More generally, by [9, Theorem 1.2], $X$ admits a $\sigma$-ample line bundle if and only if $\sigma$ has zero entropy, and in this case, any ample line bundle on $X$ is $\sigma$-ample. For this reason, the following weaker conjecture would also be interesting toward Conjecture 1.1 ([21, Conjecture 0.3]).

Conjecture 1.4. Every wild automorphism $\sigma$ of a projective variety $X$ has zero entropy.

Theorem 1.5 (1) below (part of Proposition 6.4), is also the key in giving a complete proof of Theorem 7.4, thus, together with Theorem 1.2, proving Conjecture 1.1 in dimension three, assuming Conjecture 7.3.

Theorem 1.5. Conjecture 1.4 is true if either one of the following cases occurs.

(1) $\operatorname{dim} X \leq 3$.

(2) $\operatorname{dim} X=4$, and the Kodaira dimension $\kappa(X) \geq 0$.

(3) $\operatorname{dim} X=4, \kappa(X)=-\infty$, and the irregularity $q(X) \neq 1,2$.

Acknowledgement. The authors would like to thank J. A. Chen, M. Satriano, Ch. Schnell and J. Xie for the valuable discussions, and the referee for several constructive comments to improve the paper.

\section{Preliminary Results in arbitrary dimensions}

In this section, we collect some results mostly noticed in [21]. We present them in a slightly different and expanded way. The main new ingredients are Proposition 2.3 (Fujiki and Lieberman type result) and Lemma 2.8 on the maximal rationally connected fibration (a special choice of the birational model: Chow reduction) after [17]. For instance, Proposition 2.3 can be used to prove Theorem 1.2 for $\operatorname{dim} X \leq 2$ without any detailed analysis of the geometry of ruled surfaces. See the proof of Theorem 3.1.

First of all, the following easy remark justifies our assumption on the base field.

Proposition 2.1. Let the base field $k$ be an algebraic closure of a finite field $\mathbb{F}_{q}$. Then no automorphism of any projective variety of positive dimension (over $k$ ) is wild.

Proof. Suppose the contrary that $X$ is a positive dimensional projective variety over $k$ and $\sigma$ is an automorphism of $X$ over $k$. Since $k$ is an algebraic closure of $\mathbb{F}_{q}$, both $X$ and $\sigma$ are defined over some field $k_{0}$, which is a finite extension of $\mathbb{F}_{q}$. In particular, $k_{0}$ is a finite field as well. Replacing $k_{0}$ by a finite extension if necessary, we may assume further that $X\left(k_{0}\right) \neq \emptyset$. Here $X\left(k_{0}\right)$ is the set of $k_{0}$-rational points of $X$. Since $k_{0}$ is a finite field, 
$X\left(k_{0}\right)$ is a finite set. Moreover, since $\sigma$ is defined over $k_{0}$, we have $\sigma\left(X\left(k_{0}\right)\right)=X\left(k_{0}\right)$. Since $X\left(k_{0}\right) \neq X$ from $\operatorname{dim} X>0$, it follows that $\sigma$ is not wild if $\operatorname{dim} X>0$.

Proposition 2.2. (cf. [21, Proposition 3.2, Remark 8.3]) Let $X$ be a projective variety. Suppose that an algebraic group $G \subseteq \operatorname{Aut}(X)$ acts regularly on $X$ such that $\sigma \in G$ is a wild automorphism. Then $X$ is an abelian variety and $\sigma$ is a torus translation of infinite order, with the origin of $X$ chosen in any way.

The result below is proved in [6] and [15] and generalized in [5, Theorem 1.2]. See also [14, Theorem 1.4] for a purely algebraic proof. We note that $\operatorname{Aut}_{0}(X)$ is a connected group; hence we get the triviality of its action on the (discrete) lattice $\mathrm{NS}(X) /$ (torsion) as well as its action on $\mathrm{NS}(X)_{\mathbb{R}}=\mathrm{NS}(X) \otimes_{\mathbb{Z}} \mathbb{R}$.

Proposition 2.3. (cf. [6], [15], [5], [14]) Let $X$ be a normal projective variety and we fix a big Cartier divisor class $[\omega] \in \mathrm{NS}(X)_{\mathbb{R}}$ of $X$. Then the group

$$
\operatorname{Aut}_{[\omega]}(X):=\left\{g \in \operatorname{Aut}(X) \mid g^{*}[\omega]=[\omega]\right\}
$$

is a finite extension of the identity connected component $\operatorname{Aut}_{0}(X)$ of $\operatorname{Aut}(X)$, that is, $\left[\operatorname{Aut}_{[\omega]}(X): \operatorname{Aut}_{0}(X)\right]<\infty$.

Since $\operatorname{Aut}_{0}(X)$ is an algebraic group when $X$ is projective, the above two propositions with Lemma 2.5 (2) below imply the following.

Corollary 2.4. Let $X$ be a projective variety with a wild automorphism $\sigma$. Suppose a positive power of $\sigma$ fixes a big divisor class of $X$ (this is the case when the action of $\sigma$ on the Néron Severi group $\mathrm{NS}(X)$ is of finite order). Then $X$ is an abelian variety.

We collect some basic properties of wild automorphisms in the two results below.

Lemma 2.5. (cf. [21, Lemma 3.1, Proposition 4.1, Proposition 5.1]) Let $X$ be a projective variety and let $\sigma$ be an automorphism on $X$.

(1) If $\sigma$ is wild then $X$ is smooth.

(2) $\sigma$ is wild if and only if so is $\sigma^{m}$ for some $m \geq 1$ (and hence for all $m \geq 1$ ).

(3) Suppose that $\sigma$ is a wild automorphism of $X$ and $f: X \rightarrow Y$ (resp. $g: W \rightarrow$ $X$ with $g(\operatorname{Sing}(W)) \neq X)$ is a $\sigma$-equivariant surjective morphism of projective varieties. Then $f$ (resp. $g$ ) is a smooth morphism.

(4) Suppose that $f: X \rightarrow Y$ is a $\sigma$-equivariant surjective morphism to a projective variety $Y$. If the action of $\sigma$ on $X$ is wild then so is the action of $\sigma$ on $Y$ (and hence $Y$ is smooth). 
(5) Suppose that $f: X \rightarrow Y$ is a $\sigma$-equivariant generically finite surjective morphism of projective varieties. Then the action of $\sigma$ on $X$ is wild if and only if so is the action of $\sigma$ on $Y$. Further, if this is the case, then $f: X \rightarrow Y$ is a finite étale morphism, and in particular, it is an isomorphism when $f$ is birational.

(6) If $X$ is an abelian variety and $\sigma$ is wild then $\sigma$ has zero entropy.

Proof. (1) Since Sing $X$ is stabilized by every automorphism and $\sigma$ acts as a wild automorphism on $X$, our $X$ is smooth.

For (2), if $\sigma^{m}$ stabilizes a Zariski-closed subset $Z$ of $X$, then $\sigma$ stabilizes the closed subset $\cup_{i=0}^{m-1} \sigma^{i}(Z)$ of $X$. Hence (2) is true.

(3) Let $Y_{1} \subseteq Y$ be the subset consisting of points $y \in Y$ such that the fibre $X_{y}=$ $X \times_{k} k(y)$ is not smooth. Let $Y_{2} \subseteq Y$ be the subset consisting of points $y \in Y$ such that $f: X \rightarrow Y$ is not flat at $y$. Since $X$ is smooth, both $Y_{i}$ (resp. their inverses in $X$ ) are Zariski-closed proper subsets of $Y$ (resp. $X$ ) and they are $\sigma$-stable. Hence $Y_{i}=\emptyset$. So $f$ is smooth. The case of $g$ is similar, by considering the subset of $X$ over which $g$ is non-flat or singular, each of which is a Zariski-closed subset of $W$ being different from $W$ by the generic flatness or by our additional assumption that $g(\operatorname{Sing}(W)) \neq X$.

(4) and (5) are similar (and use (3)).

(6) follows from [21, Theorem 0.2].

A smooth projective variety $V$ is a Calabi Yau manifold in the strict sense if $V$ is simply conencted, $K_{V} \sim 0$ and $H^{j}\left(V, \mathcal{O}_{V}\right)=0(0<j<\operatorname{dim} V)$.

Proposition 2.6. (cf. [21, Lemma 3.1, Corollary 4.2, Proposition 4.4, Remark 4.5]) Let $X$ be a projective variety of dimension $\geq 1$, with a wild automorphism $\sigma$. Then we have:

(1) Both the Euler Poincaré characteristic and the topological Euler number vanish: $\chi\left(\mathcal{O}_{X}\right)=0, e(X)=0$. In particular, $X$ is not rationally connected.

(2) Let $L \in \operatorname{Pic}(X)$ such that $\sigma^{*} L=L$ in $\operatorname{Pic}(X)$. Then $|L|=\emptyset$ or $L=\mathcal{O}_{X}$ in $\operatorname{Pic}(X)$. In particular, the Kodaira dimension $\kappa(X) \leq 0$.

(3) Suppose that $\kappa(X)=0$. Then $K_{X} \sim_{\mathbb{Q}} 0$; the Beauville-Bogomolov (minimal split) finite étale cover $\tilde{X}$ of $X$ is a product of an abelian variety $A$ of dimension $\geq 0$ and a few copies of Calabi Yau manifolds $C_{i}$ of odd dimension $\geq 3$ and in the strict sense; and a positive power of $\sigma$ lifts to a diagonal action on $\widetilde{X}=A \times \prod_{i} C_{i}$ whose action on each factor is wild.

Proof. (1) See [21, Proposition 4.4, Remark 4.5].

If (2) is false, $|L| \simeq \mathbb{P}^{N}$ with $N \geq 1$. It follows that the action of $\sigma$ on $|L|$ has a fixed point, say $D \in|L|$. Then $\sigma(D)=D$, contradicting the fact that $\sigma$ is wild. 
(3) We have $K_{X} \sim_{\mathbb{Q}} D$ for some effective $\mathbb{Q}$-divisor (which is unique). Thus $D$ is $\sigma$-stable. Hence $D=0$, and $K_{X} \sim_{\mathbb{Q}} 0$. Let $\widetilde{X} \rightarrow X$ be the Beauville-Bogomolov covering such that $\widetilde{X}$ is the product of an abelian variety $A$, hyperkähler manifolds $H_{i}$, and Calabi Yau manifolds $C_{j}$ in the strict sense. Replacing the cover $\tilde{X}$ by the minimal splitting cover in [2, §3], we can lift $\sigma$ to an action on $\widetilde{X}$ so that $\widetilde{X} \rightarrow X$ is $\sigma$-equivariant. Hence the action of $\sigma$ on $\tilde{X}$ is also wild; further, $\sigma$ (replaced by a positive power) splits as diagonal actions on the factors $A, H_{i}$ and $C_{j}$ (cf. [2, §3]), which are still wild by Lemma 2.5, and hence these factors have the vanishing Euler Poincare characteristic by (1). Thus the $H_{i}$ does not appear, and the $C_{j}$ are Calabi Yau manifolds of odd dimension $(\geq 3)$ in the strict sense. This proves the proposition.

The result below uses the standard results on subvarieties of a complex tori in [24].

Lemma 2.7. (cf. [21, Corollary 4.3, Proposition 5.1]) Let $X$ be a (smooth) projective variety with a wild automorphism $\sigma$, let $A$ be an abelian variety and let $f: X \rightarrow A$ be a $\sigma$-equivariant morphism. Then the image $Y:=f(X)$ is a subtorus of $A$ and $f: X \rightarrow Y$ is a smooth surjective morphism. In particular, the albanese map $\operatorname{alb}_{X}: X \rightarrow A=\operatorname{Alb}(X)$ is a surjective smooth morphism with connected fibres.

Proof. It is known that every subvariety of an abelian variety, like $Y=f(X)$ of $A$, has Kodaira dimension $\kappa(Y) \geq 0$ and equality holds if and only if $Y$ is a subtorus (after choosing a new origin for $A$ ); further, if the inequality is strict then there is a subtorus $B \subset A$ acting on $Y$ such that $Y \rightarrow Y / B$ is birational to the Iitaka fibration, see [24, Theorem 10.9, Corollary 10.12]. Now our $\sigma$ acts on $Y$ and descends to a birational automorphism $\left.\sigma\right|_{Y / B}$ on the base of this Iitaka fibration. By the finiteness of the pluricanonical representation [24, Theorem 14.10], $\left.\sigma\right|_{Y / B}$ is of finite order. Hence a sum of finitely many fibres of $X \rightarrow Y / B$ is $\sigma$-stable. Since $\sigma$ is wild, this sum equals $X$ itself. Hence $Y / B$ is a point, so $Y$ equals $B$, a subtorus of $A$. The surjective morphism $X \rightarrow Y=f(X)$ is smooth by Lemma 2.5 .

For the final assertion, note that $\operatorname{alb}_{X}: X \rightarrow \operatorname{Alb}(X)$ is $\operatorname{Aut}(X)$ - and hence $\sigma$ equivariant, and $\operatorname{Alb}(X)$ is generated by the image $\operatorname{alb}_{X}(X)$ (an abelian variety), so $\operatorname{alb}_{X}(X)=\operatorname{Alb}(X)$. Let $g: X \rightarrow W$ be the Stein factorization of $\operatorname{alb}_{X}$. Since $\operatorname{alb}_{X}$ is $\sigma$-equivariant, so is $g$. By Lemma 2.5, the induced morphisms $\left.\sigma\right|_{W}$ and $\left.\sigma\right|_{\operatorname{Alb}(X)}$ are both wild, $W$ is smooth, and the induced morphism $X \rightarrow W$ is smooth while $W \rightarrow \operatorname{Alb}(X)$ is étale. Hence $W$ is also an abelian variety. In fact, the surjective étale morphism $W \rightarrow \operatorname{Alb}(X)$ is an isomorphism by the universality of the albanese morphism. This proves the lemma. 
A maximal rationally connected (MRC) fibration on a normal projective variety has general fibres $F$ rationally connected in the sense of Campana and Kollár-Miyaoka-Mori (i.e., every two points on $F$ are connected by an irreducible rational curve) and it is 'maximal' among such (rational) fibrations. See eg. [11, Definition 5.3, Theorem 5.4].

Lemma 2.8. Let $X$ be a (smooth) projective variety of positive dimension, with a wild automorphism $\sigma$. Suppose that $X$ is uniruled. Then we can choose the maximal rationally connected (MRC) fibration $X \rightarrow Y$ to be a well defined $\sigma$-equivariant surjective smooth morphism with $0<\operatorname{dim} Y<\operatorname{dim} X$. Further, the action of $\sigma$ on $Y$ is also wild.

Proof. By Nakayama [17, Proposition 4.14 or Theorem 4.18], we can choose MRC $X--\rightarrow$ $Y$ to be a (unique) special Chow reduction with the graph $\Gamma=\Gamma_{X / Y}$ equi-dimensional over $Y$. Especially, $\sigma$ on $X$ descends to an automorphism $\sigma_{Y}$ on $Y$. The natural birational map $\Gamma \rightarrow X$ is $\sigma$-equivariant, and hence it is an isomorphism; see Lemma 2.5. Thus we may assume that $X=\Gamma \rightarrow Y$ is a well-defined surjective morphism. Hence it is smooth by the same Lemma 2.5. Since $X$ is not rationally connected by Proposition 2.6, $Y$ is not a point. Since $X$ is uniruled, $\operatorname{dim} Y<\operatorname{dim} X$. The action of $\sigma$ on $Y$ is wild by Lemma 2.5. This proves the lemma.

The following conjecture is known in dimension up to three (cf. [12, §3.13] and the references therein).

Conjecture 2.9. (Weak abundance conjecture) A projective variety is uniruled (i.e., covered by rational curves) if and only if it has negative Kodaira dimension.

Proposition 2.10. Let $X$ be a (smooth) projective variety of dimension $\geq 1$, with a wild automorphism $\sigma$. Assume either $\operatorname{dim} X \leq 3$ or the weak abundance conjecture in dimension $\leq n$. Then we can choose the maximal rationally connected (MRC) fibration to be a $\sigma$-equivariant surjective smooth morphism $f: X \rightarrow Y$ (with every fibre a rationally connected variety of dimension $\geq 0$ ); $\operatorname{dim} Y>0$; the action of $\sigma$ on $Y$ is wild (and hence $Y$ is smooth); $K_{Y} \sim_{\mathbb{Q}} 0$; and the Beauville-Bogomolov finite étale cover of $Y$ is a product of an abelian variety of dimension $\geq 0$ and a few copies of Calabi-Yau manifolds of odd dimension $\geq 3$ in the strict sense.

Proof. By Proposition 2.6, we have the Kodaira dimension $\kappa(X) \leq 0$. If $\kappa(X)=0$, the proposition follows from Proposition 2.6.

Thus we may assume that $\kappa(X)<0$. Then $X$ is uniruled, by our assumption. Using Lemma 2.8, we can choose the maximal rationally connected (MRC) fibration $X \rightarrow Y$ to be a well defined $\sigma$-equivariant surjective smooth (non-trivial) morphism. By Lemma 2.5 the induced action $\left.\sigma\right|_{Y}$ on $Y$ of $\sigma$ is also wild. Thus every fibre of $X \rightarrow Y$ is rationally 
connected, since $\left.\sigma\right|_{Y}$ fixes the locus on $Y$ (a non-dense proper subset of $Y$ ) over which the fibres are not rationally connected. $Y$ is not uniruled by [7, Corollary 1.4]. Hence the Kodaira dimension $\kappa(Y) \geq 0$ by the assumed weak abundance conjecture. Now the proposition follows from Proposition 2.6.

Lemma 2.11. Let $X$ be a (smooth) projective variety with a wild automorphism $\sigma$, let $A$ be an abelian variety and let $f: X \rightarrow A$ be a $\sigma$-equivariant surjective morphism with connected fibres of positive dimension. Suppose that a positive power $\sigma^{s}$ of $\sigma$ fixes the class of a big divisor $D$ on $A$ in $\operatorname{NS}(A)_{\mathbb{R}}=\mathrm{NS}(A) \otimes_{\mathbb{Z}} \mathbb{R}$ (this holds if $\operatorname{dim} A=1$ or a positive power of $\sigma$ is a translation on $A)$. Then $-K_{F}$ is not a big divisor, where $F$ is a general fibre of $f$.

Proof. By Lemma 2.5, $f$ is a smooth morphism. Suppose the contrary that $-K_{F}$ is a big divisor. Then $-K_{X}$ is relatively big over $A$. Thus $E:=-K_{X}+m f^{*} D$ is a big divisor for some $m>>1$, see (1) in the proof of [3, Lemma 2.5]. Now $\sigma^{s}$ fixes the big class $[E]$. Thus, by Corollary $2.4, X$ is an abelian variety and hence $K_{F}=\left.K_{X}\right|_{F}=0$, a contradiction. This proves the lemma. Indeed, for the claim in the bracket of the statement of the lemma, if $\operatorname{dim} A=1$, then $\sigma^{12}$ is a translation on $A$; if a positive power $\sigma^{t}$ of $\sigma$ is a translation on $A$, then it fixes every ample divisor class on $A$.

The lemma below was proved in [10].

Lemma 2.12. (cf. [10, Lemma 2.8]) Let $\sigma$ be a wild automorphism of an abelian variety A. Let $H \leq \operatorname{Aut}_{\text {variety }}(A)$ be a finite subgroup centralized by $\sigma: \sigma h=h \sigma$ for all $h \in H$. Then $H$ consists of translations of $A$.

A projective variety $X$ is called a $\mathbb{Q}$-torus if it has an abelian variety $A_{1}$ as an étale finite cover (or equivalently it is the quotient of an abelian variety $A_{2}$ by a finite group acting freely on $A_{2}$ ).

Proposition 2.13. Let $X$ be a projective $\mathbb{Q}$-torus with a wild automorphism $\sigma$. Then $X$ is an abelian variety.

Proof. Let $A \rightarrow X$ be the minimal splitting cover of $X$ in $[2, \S 3]$. Then $\sigma$ lifts to an automorphism on $A$, also denoted as $\sigma$. Note that the $\sigma$ on $A$ normalizes $H:=\operatorname{Gal}(A / X)$. Hence $\sigma^{r !}$ centralizes every element of $H$, where $r:=|H|$. Since $\sigma^{r !}$ is still wild by Lemma 2.5, $H$ consists of translations by Lemma 2.12. Hence $H=\left\{i d_{A}\right\}$ by the minimality of $A \rightarrow X$. Therefore $X=A$ and $X$ is an abelian variety.

Lemma 2.14. Let $X$ be a smooth projective variety, $C$ a smooth irreducible projective curve, and $f: X \rightarrow C$ a smooth morphism. Let $\eta$ be the generic point of $C$ and $X_{\eta}=$ 
$f^{-1}(\eta)$ the generic fibre. Suppose $\operatorname{Pic}\left(X_{\eta}\right) \simeq \operatorname{NS}\left(X_{\eta}\right)$ (this is the case when $\operatorname{Pic}^{0}\left(X_{\eta}\right)=0$, eg. when the geometric generic fibre $X_{\bar{\eta}}$ is rationally connected). Then $\left.\operatorname{NS}(X)\right|_{X_{\eta}}=$ $\mathrm{NS}\left(X_{\eta}\right)$ and the kernel of the surjection

$$
\left.\mathrm{NS}(X)_{\mathbb{Q}} \rightarrow \mathrm{NS}(X)_{\mathbb{Q}}\right|_{X_{\eta}}=\mathrm{NS}\left(X_{\eta}\right)_{\mathbb{Q}}
$$

is spanned by $\mathbb{Q}[F]$, where $[F]$ is the class of closed fibres of $f$.

Proof. Note that algebraic equivalence is preserved under morphisms (hence under restrictions). Thus $\left.\mathrm{NS}(X)\right|_{X_{\eta}} \subseteq \mathrm{NS}\left(X_{\eta}\right)$. Let $D$ be a prime divisor on $X_{\eta}$ and let $\bar{D}$ be the Zariski closure of $D$ in $X$. Then $\bar{D}$ is a divisor on $X$ and $D=\left.\bar{D}\right|_{X_{\eta}}$ as divisors. This implies $\left.\mathrm{NS}(X)\right|_{X_{\eta}}=\mathrm{NS}\left(X_{\eta}\right)$.

Let $L, M \in \operatorname{Pic}(X)$ such that $\left.\left.L\right|_{X_{\eta}} \cong M\right|_{X_{\eta}}$ in $\operatorname{Pic}\left(X_{\eta}\right)$. It suffices to show that $L=M \otimes f^{*} N$ for some $N \in \operatorname{Pic}(C)$.

Since $\left.\left.L\right|_{X_{\eta}} \cong M\right|_{X_{\eta}}$, it follows that

$$
h^{0}\left(X_{\eta},\left.\left(L \otimes M^{-1}\right)\right|_{X_{\eta}}\right)=1, h^{0}\left(X_{\eta},\left.\left(L \otimes M^{-1}\right)^{-1}\right|_{X_{\eta}}\right)=1 .
$$

Then by the upper-semicontinuity theorem,

$$
h^{0}\left(X_{y},\left.\left(L \otimes M^{-1}\right)\right|_{X_{y}}\right) \geq 1, h^{0}\left(X_{y},\left.\left(L \otimes M^{-1}\right)^{-1}\right|_{X_{y}}\right) \geq 1
$$

for all closed fibres $X_{y}$. Since $X_{y}$ is smooth projective, it follows that $\left.\left(L \times M^{-1}\right)\right|_{X_{y}} \simeq \mathcal{O}_{X_{y}}$ and therefore $h^{0}\left(X_{y},\left.\left(L \times M^{-1}\right)\right|_{X_{y}}\right)=1$ for all closed fibres $X_{y}$. Then, since the morphism $f$ is smooth, hence flat, the sheaf $N:=f_{*}\left(L \otimes M^{-1}\right)$ is an invertible sheaf with base change property $f_{*}\left(L \otimes M^{-1}\right) \otimes \kappa(y) \simeq H^{0}\left(X_{y},\left.\left(L \times M^{-1}\right)\right|_{X_{y}}\right)$ by Grauert's theorem. Since $\left.\left(L \otimes M^{-1}\right)\right|_{X_{y}} \simeq \mathcal{O}_{X_{y}}$ is globally generated, by Nakayama's lemma, the natural morphism

$$
f^{*} N:=f^{*} f_{*}\left(L \otimes M^{-1}\right) \rightarrow L \otimes M^{-1}
$$

is then surjective. Since both sides are invertible sheaves, it follows that $f^{*} N \simeq L \otimes M^{-1}$, i.e., $L=M \otimes f^{*} N$ as desired.

Proposition 2.15. Let $X$ be a projective variety over $\mathbb{C}$ of $\operatorname{dim} X=n$ with a wild automorphism $\sigma$. Then the albanese morphism $\operatorname{alb}_{X}: X \rightarrow \operatorname{Alb}(X)$ is a smooth surjective morphism with connected fibres and hence $0 \leq q(X)=\operatorname{dim} \operatorname{Alb}(X) \leq n$. If $q(X)=n$, then $X=\operatorname{Alb}(X)$, an abelian variety; if $q(X) \geq n-1$, then $\sigma$ has zero entropy.

Proof. By Lemma 2.7, $\operatorname{alb}_{X}: X \rightarrow \operatorname{Alb}(X)$ is a smooth surjective morphism with connected fibres. Assume that $q(X)=n-1$. Then, since the fibre of alb ${ }_{X}$ is one dimensional,

$$
d_{1}\left(\left.\sigma\right|_{X}\right)=d_{1}\left(\left.\sigma\right|_{\operatorname{Alb}(X)}\right)=1
$$


by the product formula in [4] and the fact that $\left.\sigma\right|_{\operatorname{Alb}(X)}$ is a wild automorphism of an abelian variety and hence has zero entropy (cf. Lemma 2.5). The rest of the proposition is clear.

Proposition 2.16. Let $X$ be a projective variety over $\mathbb{C}$. Suppose $\sigma$ is a wild automorphism of $X$. Put

$$
A:=\oplus_{k \geq 0} H^{2 k+1}(X, \mathbb{Z}) /(\text { torsion }), \quad B:=\oplus_{k \geq 0} H^{2 k}(X, \mathbb{Z}) /(\text { torsion }) .
$$

Then the set of complex eigenvalues of $\left.\sigma^{*}\right|_{A}$ and the set of complex eigenvalues of $\left.\sigma^{*}\right|_{B}$ coincide counted with multiplicities.

Proof. By Lemma 2.5, $X$ is smooth. By Proposition 2.6, the topological Euler number $e(X)=0$. Thus $A$ and $B$ have the same rank, say $r>0$. Let $\left\{a_{i}\right\}_{i=1}^{r}$ be the set of complex eigenvalues of $\sigma^{*}$ on $A$ counted with multiplicities and $\left\{b_{i}\right\}_{i=1}^{r}$ be the set of complex eigenvalues of $\sigma^{*}$ on $B$ counted with multiplicities.

Since $\sigma^{n}$ is wild, the topological Lefschetz number of $\sigma^{n}$ is 0, i.e.,

$$
\sum_{i} a_{i}^{n}=\sum_{i} b_{i}^{n}
$$

for all $n$, in particular, for all $n=1,2, \ldots, r$. By Newton's identities, this implies that the elementary symmetric polynomials in $a_{1}, \ldots, a_{r}$ coincide with the elementary symmetric polynomials in $b_{1}, \ldots, b_{r}$. Hence the monic polynomial with roots $\left\{a_{i}\right\}_{i=1}^{r}$ counted with multiplicities and the one with roots $\left\{b_{i}\right\}_{i=1}^{r}$ counted with multiplicities coincide. Thus $\left\{a_{i}\right\}_{i=1}^{r}=\left\{b_{i}\right\}_{i=1}^{r}$ counted with multiplicities.

\section{Preliminary Results in LOWER Dimensions}

Theorem 3.1 below was proved in [21, §6]. Our Lemma 2.11 (and Proposition 2.3 implicitly) helps skipping the detailed analysis of ruled surfaces; compare with the proof of [21, Lemma 6.2].

Theorem 3.1. (cf. $[21, \S 6]$ ) Let $X$ be a projective variety with a wild automorphism $\sigma$. Suppose that $\operatorname{dim} X \leq 2$. Then $X$ is an abelian variety.

Proof. Assume to the contrary that $X$ is not an abelian variety. Then by Proposition 2.10 and Proposition 2.13, it follows that $\operatorname{dim} X=2$ and $X$ admits a smooth fibration $f: X \rightarrow Y$ with fibres $F$ smooth rational curve and $Y$ an elliptic curve. But then a general fibre $F$ of $f$ satisfies $F \cong \mathbb{P}^{1}$ has ample $-K_{F}$, contradicting Lemma 2.11.

Theorem 1.2 for threefolds will follow from Proposition 3.2 below and Propositions 4.1, 5.2 and 6.4 in subsequent sections. 
Proposition 3.2. Let $X$ be a projective variety of dimension three with a wild automorphism $\sigma$. Suppose that $X$ is neither an abelian variety, nor a Calabi-Yau manifold. Then $X$ is uniruled and the maximal rationally connected (MRC) fibration can be chosen to be a $\sigma$-equivariant surjective smooth morphism $f: X \rightarrow Y$ and coincides with the albanese map $\operatorname{alb}_{X}: X \rightarrow \operatorname{Alb}(X)=\operatorname{Alb}(Y)$, such that $\sigma$ acts on $Y$ as a wild automorphism and one of the following cases occurs.

(1) The irregularity $q(X)=1$. In this case, $Y$ is an elliptic curve, $\sigma$ acts on $Y$ as a translation of infinite order and every closed fibre $X_{y}$ over $y \in Y$ is a smooth rational surface.

(2) $q(X)=2$. In this case, $Y$ is an abelian surface, $\sigma$ acts on $Y$ as a wild automorphism, and every closed fibre $X_{y}$ over $y \in Y$ is a smooth rational curve: $X_{y} \cong \mathbb{P}^{1}$.

Proof. Since $X$ is neither an abelian threefold, nor a Calabi-Yau threefold, by virtue of Proposition 2.10 and Proposition 2.13, $\mathrm{X}$ is uniruled and the MRC $f: X \rightarrow Y$ is a $\sigma$-equivariant surjective smooth morphism with $Y$ an abelian variety of dimension 1 or 2 and every fibre $F$ a smooth rational variety of dimension 2 or 1 , respectively. Here we use the fact that a rationally connected variety of dimension at most two is a rational variety.

Since $Y=\operatorname{Alb}(Y)$, there is a morphism $g: \operatorname{Alb}(X) \rightarrow Y$ such that $g \circ \operatorname{alb}_{X}=f$ by the universal property of the albanese map $\operatorname{alb}_{X}$. Since $f$ is surjective with connected fibres, so is $g$. Let $X_{y}(y \in Y)$ be any closed fibre of $f$. Then $\operatorname{alb}_{X}\left(X_{y}\right)$ is a point for each $X_{y}$, because $X_{y}$ is rationally connected and any abelian variety contains no rational curves. Thus $g$ is a finite morphism (with connected fibres), so it is an isomorphism by the normality (and indeed smoothness) of $\mathrm{Alb}(X)$ and $Y$. This proves the proposition.

The next result is certainly known at least over $\mathbb{C}$ (cf. [22, Table 3.1]). For the sake of completeness and its own interest, we shall give a proof which is vailid over any algebraically closed field of characteristic $\neq 2$.

Proposition 3.3. Let $\pi: S \rightarrow E$ be a relatively minimal elliptic ruled surface over an algebraically closed field of characteristic $\neq 2$. Then the Iitaka D-dimension $\kappa\left(S,-K_{S}\right) \geq$ 0 .

Proof. We write $S=\mathbb{P}(\mathcal{E})=\operatorname{Proj}\left(\oplus_{n \geq 0} \operatorname{Sym}^{n}(\mathcal{E})\right)$ where $\mathcal{E}$ is a normalized rank 2 locally free sheaf on the elliptic curve $E$. Then we have an exact sequence

$$
0 \rightarrow \mathcal{O}_{E} \rightarrow \mathcal{E} \rightarrow \mathcal{L} \rightarrow 0
$$


for some invertible sheaf $\mathcal{L}$ on $E$. Let $C_{0}$ be the section corresponding to this surjection. Then, with respect to $\mathcal{O}_{S}(1)$ defined by $\mathcal{E}$, one has $\mathcal{O}_{S}(1)=\mathcal{O}_{S}\left(C_{0}\right)$ and

$$
-K_{S}=\mathcal{O}_{S}(2)-\pi^{*} \operatorname{det} \mathcal{E}=\mathcal{O}\left(2 C_{0}\right)-\pi^{*} \operatorname{det} \mathcal{E}=\mathcal{O}\left(2 C_{0}\right)-\pi^{*} \mathcal{L}
$$

in $\operatorname{Pic}(S)$ by the canonical bundle formula.

If $\mathcal{E}$ is decomposable, then $\operatorname{deg} \mathcal{L} \leq 0$ and therefore $\mathcal{E}=\mathcal{O}_{E} \oplus \mathcal{L}$. Corresponding to the projection $\mathcal{E} \rightarrow \mathcal{O}_{E}$, we have a section $C_{1}$ such that $\mathcal{O}_{S}\left(C_{1}\right)=\mathcal{O}_{\mathbb{P}(\mathcal{E})}(1)-\pi^{*} \operatorname{det} \mathcal{E}$ in $\operatorname{Pic}(S)$. Hence $-K_{S}$ is linearly equivalent to an effective divisor $C_{0}+C_{1}$.

If $\mathcal{E}$ is indecomposable of degree 0 , then $\mathcal{E}$ is the non-trivial extension of the form

$$
0 \rightarrow \mathcal{O}_{E} \rightarrow \mathcal{E} \rightarrow \mathcal{O}_{E} \rightarrow 0
$$

and therefore $-K_{S} \cong \mathcal{O}_{S}(2)$. Hence $-K_{S}$ is linearly equivalent to the effective divisor $2 C_{0}$, where $C_{0}$ is the section given by the exact sequence above.

It remains to consider the case where $\mathcal{E}$ is indecomposable of degree 1. Our proof below is inspired by [23].

Consider the product surface $\mathbb{P}^{1} \times E$ and its automorphism subgroup $G$ generated by $\tau_{1}$ and $\tau_{2}$ defined by

$$
\tau_{1}:(t, s) \mapsto(-t, s+a), \quad \tau_{2}:(t, s) \mapsto\left(t^{-1}, s+b\right)
$$

where $\langle a, b\rangle$ is the group of two torsion points of $E$. Then $G$ acts freely on $\mathbb{P}^{1} \times E$ and we have a smooth projective surface $S=\left(\mathbb{P}^{1} \times E\right) / G$. The two projections on $\mathbb{P}^{1} \times E$ induce on $S$ a relatively minimal elliptic fibre space structure $p_{1}: S \rightarrow \mathbb{P}^{1}=\mathbb{P}^{1} / G$ and a relatively minimal elliptic ruled surface structure $p_{2}: S \rightarrow E \simeq E / G$.

We are going to show that $\kappa\left(S,-K_{S}\right) \geq 0$ and $p_{2}: S \rightarrow E \simeq E / G$ is the unique minimal elliptic ruled surface over $E$ corresponding to an indecomposable locally free sheaf of rank 2 and degree 1.

Observe that $p_{1}$ has exactly three multiple fibres of type ${ }_{2} I_{0}$. Hence, by the canonical bundle formula for an elliptic surface applied for $p_{1}$, it follows that $-2 K_{S}$ is linearly equivalent to $F$, any smooth fibre of $p_{1}$. In particular, $\kappa\left(S,-K_{S}\right) \geq 0$.

Now it suffices to show that $p_{2}: S \rightarrow E$ is a ruled surface corresponding to an indecomposable locally free shaef $\mathcal{E}$ of rank 2 and degree 1 . Recall that such a minimal elliptic ruled surface is unique from $E$ and does not depend on the choice of such sheaf $\mathcal{E}$, and this is also the unique case so that $\left(C_{0}^{2}\right)>0$, where $C_{0}$ is the section of $p_{2}$ as defined at the beginning of this proof.

Observe that $\left.\operatorname{deg} p_{2}\right|_{F_{t}}=2$ or 4 for any fibre $F_{t}$ of $p_{1}$ with reduced structure. Then the section $C_{0}$ of $p_{2}$ is an elliptic curve, but not in the fibre of $p_{1}$ (whose multiplicity is at 
most 2). Thus $\left(F \cdot C_{0}\right)>0$ and hence $\left(C_{0}^{2}\right)>0$ by

$$
0=\operatorname{deg} K_{C_{0}}=\left(\left(K_{S}+C_{0}\right) \cdot C_{0}\right)=-\left(F \cdot C_{0}\right) / 2+\left(C_{0}^{2}\right) .
$$

This proves the result.

Lemma 3.4. Let $\pi: S \rightarrow E$ be a relatively minimal ruled surface over an elliptic curve $E$ defined over an algebraically closed field of characteristic $\neq 2$. Then $\kappa\left(S,-K_{S}\right) \geq 0$ and one of the following cases occurs.

(1) $\kappa\left(S,-K_{S}\right)=2$. In this case for all $t>>1$ and sufficiently divisible, the base locus of $\left|-t K_{S}\right|$ has a 1-dimensional irreducible component.

(2) $\kappa\left(S,-K_{S}\right)=1$. In this case, $\left|-t K_{S}\right|$ is base point free for some $t \geq 1$ and it defines a relatively minimal elliptic fibration $S \rightarrow \mathbb{P}^{1}$.

(3) $\kappa\left(S,-K_{S}\right)=0$. In this case, there is an integer $m>0$ such that $\left|-m K_{S}\right|=\{D\}$ for a non-zero effective divisor $D$ on $S$.

Proof. Note that $\kappa\left(S,-K_{S}\right) \geq 0$ by Proposition 3.3 or [22, Table 3.1] (when $k=\mathbb{C}$ ).

Let $-K_{S}=P+N$ be the Zariski-decomposition, where $P$ is nef, $P . N=0, N=\sum a_{i} N_{i}$ is effective and has negative intersection matrix $\left(N_{i} \cdot N_{j}\right)$. Note that $\kappa\left(S,-K_{S}\right)=\kappa(S, P)$, and the integral part of $t N$ is contained in the fixed part of $\left|-t K_{S}\right|$ for all $t \geq 1$.

If $\kappa\left(S,-K_{S}\right)=2$, then $-K_{S}$ and hence $P$ are big. So $P$ is nef and big, and $P^{2}>0$. Thus $0=\left(-K_{S}\right)^{2}=P^{2}+N^{2}>N^{2}$ implies that $N \neq 0$. This is Case(1).

Now we may assume that $\kappa\left(S,-K_{S}\right)=0$ or 1 . Hence $P$ is not big, so $P^{2}=0$. Thus $0=\left(-K_{S}\right)^{2}=P^{2}+N^{2}=N^{2}$ implies $N=0$ (the zero divisor). Namely, $-K_{S}=P$ is nef (but not big).

Suppose that $\kappa\left(S,-K_{S}\right)=0$. Then the statement in Case (3) is clear, because $-K_{S}$, hence $K_{S}$, is not numerically trivial.

Suppose that $\kappa\left(S,-K_{S}\right)=1$. Since $-K_{S}$ is nef and $\left(-K_{S}\right)^{2}=0$, it follows that $\left|-t K_{S}\right|$ is base point free for $t>>1$ and sufficiently divisible. The adjunction formula for fibre, implies then that $\left|-t K_{S}\right|$ defines an elliptic fibration $f: S \rightarrow \mathbb{P}^{1}$ (with fibres of the ruling $S \rightarrow E$ all horizontal to $f$ ). This proves the lemma.

\section{Proof of Theorem 1.2 When $q=2$}

Proposition 4.1. The case $q(X)=2$ in Proposition 3.2 does not occur. (Here our $X$ is defined over $k=\mathbb{C}$, though the argument works over any field $k=\bar{k}$ with char $k=0$.)

Proof. Suppose the contrary that the case $q(X)=2$ in Proposition 3.2 occurs and we shall derive a contradiction.

For the $\sigma$-equivariant fibration $f: X \rightarrow Y$ there, let $X_{y}$ be a fibre over $y \in Y$. Then $X_{y} \cong \mathbb{P}^{1}$. As in [21, Theorem 7.2], write the wild automorphism $\sigma=T_{b} \circ \alpha$ on $Y$ where 
$T_{b}$ is a translation on the abelian surface $Y$ and $\alpha: Y \rightarrow Y$ is a group automorphism such that the endomorphism $\beta=\alpha-\mathrm{id}_{Y}$ is nilpotent.

If $\beta=0$ then $\sigma=T_{b}$ and it is a translation; by Lemma 2.11, the anti-canonical divisor $-K_{X_{y}}$ of a general fibre $X_{y}$, is not big, which contradicts that $X_{y} \cong \mathbb{P}^{1}$ has ample anti-canonical divisor.

Thus we may assume that $\beta \neq 0$. Let $B$ be a connected component of Ker $\beta$. Then $B$ is a nontrivial proper subtorus of the 2-torus $Y$, and hence an elliptic curve. Thus $\sigma$ permutes the coset of $E:=Y / B$, an elliptic curve. Hence the quotient map $Y \rightarrow E$ is $\sigma$-equivariant. Since the action of $\sigma$ on $Y$ is wild, so is the action of $\sigma$ on $E$, see Lemma 2.5. Hence $\sigma$ is a translation of infinite order, see [21, Remark 8.3].

Consider the $\sigma$-equivariant fibration $g: X \rightarrow E$, which is the composition $X \rightarrow Y \rightarrow$ $E=Y / B$. It is a smooth fibration (cf. Lemma 2.5) with each fibre $X_{e}$ over $e \in E$ a relatively minimal ruled surface over the elliptic curve $Y_{e}$ (the fibre of $Y \rightarrow E$ over $e \in E)$, noting that every fibre of $X \rightarrow Y$ is isomorphic to $\mathbb{P}^{1}$.

For $-K_{X_{e}}=\left.\left(-K_{X}\right)\right|_{X_{e}}$, note that $h^{0}\left(X_{e},\left.\mathcal{O}\left(-m K_{X}\right)\right|_{X_{e}}\right)$ and hence $\kappa\left(X_{e},-K_{X_{e}}\right)$ are upper semi-continuous as functions in $e \in E$. The subsets of points of $E$ over which these functions obtain larger values, are Zariski closed subsets and $\sigma$-stable. Since $\sigma$ is wild, we may assume that these functions are all constants as functions in $e \in E$. Since $g: X \rightarrow E$ (a smooth curve) is flat and by Grauert's theorem, $g_{*} \mathcal{O}\left(-m K_{X}\right)$ is locally free and we have the following natural isomorphism for all $m \geq 0$ and all (closed) point $e \in E$ (with residue field $k(e)=k$ ):

$$
g_{*} \mathcal{O}\left(-m K_{X}\right) \otimes k(e) \cong H^{0}\left(X_{e},\left.\mathcal{O}\left(-m K_{X}\right)\right|_{X_{e}}\right) .
$$

For $m>>1$ and sufficiently divisible, consider the rational map

$$
h: X \rightarrow W=\mathbb{P}\left(g_{*} \mathcal{O}\left(-m K_{X}\right)\right)
$$

associated to the sheaf homomorphism

$$
g^{*} g_{*} \mathcal{O}\left(-m K_{X}\right) \rightarrow \mathcal{O}\left(-m K_{X}\right) .
$$

Since $\sigma^{*}\left(-K_{X}\right)=-K_{X}$, there is a natural action of $\sigma$ on $W$ compatible with that on $X$.

If $S=X_{e}$ has $\kappa\left(S,-K_{S}\right)=2$ or 0 , then Lemma 3.4 and the isomorphism ( $*$ ) above imply that the base locus $\mathrm{Bs}\left|-m K_{S}\right|$ is 1-dimensional for some $m>>1$ and divisible, whose union

$$
\cup_{e \in E} \operatorname{Bs}\left|-m K_{X_{e}}\right|=\operatorname{Supp} \operatorname{Coker}\left(g^{*} g_{*} \mathcal{O}\left(-m K_{X}\right) \rightarrow \mathcal{O}\left(-m K_{X}\right)\right)
$$

is a $\sigma$-stable Zariski-closed proper subset of $X$, a contradiction.

If $S=X_{e}$ has $\kappa\left(S,-K_{S}\right)=1$, then Lemma 3.4 and the isomorphism (*) above imply that $\left|-m K_{S}\right|$ defines an elliptic fibration $S \rightarrow \mathbb{P}^{1}$ for some $m>>1$ and divisible, and 
$h: X \rightarrow W$ is a well-defined morphism. Thus $W$ is a ruled surface, covered by rational curves $h\left(X_{e}\right)$, and we have a $\sigma$-equivariant fibration $h: X \rightarrow W$. But the action of $\sigma$ on $W$ is also wild by Lemma 2.5 , and hence $W$ must be an abelian surface by $[21, \S 6]$ or Theorem 3.1. This is a contradiction. This proves the proposition.

\section{Proof of Theorem 1.2 When $q=1$}

We begin with:

Lemma 5.1. Let $S$ be a smooth projective geometrically rational surface over a field $k$ (not necessarily algebraically closed) and $\Lambda:=\mathrm{NS}(S / k)$, the Néron-Severi group of $S$ over the field $k$. Let $g \in \operatorname{Aut}(X / k)$ be an automorphism of $S$ over $k$. Assume that $g$ is of zero entropy in the sense that the spectral radius of $\left.g^{*}\right|_{\Lambda}$ is one, and that $\left.g^{*}\right|_{\Lambda}$ is of infinite order. Then we have:

(1) There is a nonzero pseudo-effective $\mathbb{Q}$-Cartier divisor $v \in \Lambda_{\mathbb{Q}}:=\Lambda \otimes_{\mathbb{Z}} \mathbb{Q}$ such that $g^{*}(v) \sim_{\mathbb{Q}} v$. Moreover, the ray $\mathbb{Q}_{>0} v$ of such $v$ is unique.

(2) $v$ is nef and $v^{2}=0$.

(3) Replacing $v$ by a multiple, we have

$$
K_{S}+v \sim_{\mathbb{Q}} N(S):=\sum_{i} a_{i} N_{i}
$$

Here if $N(S) \neq 0$, then $N(S)$ is an effective divisor with $N_{i}$ integral curves, the intersection matrix $\left(N_{i} . N_{j}\right)$ is negative definite and $g^{*}(N(S))=N(S)$. The $v$ in (1) with the extra condition (*) above is unique up to $\mathbb{Q}$-linear equivalence.

Proof. Since $\Lambda$ is hyperbolic, (1) and (2) are proved in the exactly same way as in [19, Theorem 2.1]

For (3), by replacing $v$ by a positive multiple, we may assume that $v \in \Lambda$ is (represented by) an integral Cartier divisor.

First note that $K_{S} \cdot v=0$. Otherwise, $\left(K_{S}+t v\right)^{2}>0$ for some integer $t$ and $g^{*}\left(K_{S}+\right.$ $t v)=K_{S}+t v$. Since $\left(K_{S}+t v\right)^{\perp}(\subset \Lambda)$ is negative definite, it follows that $\left.g^{*}\right|_{\Lambda}$ is of finite order, a contradiction.

By the Serre duality, we also have

$$
h^{2}\left(K_{S}+v\right)=h^{0}(-v)=0
$$

as $v$ is nef and $v \neq 0$. Hence, by the Riemann-Roch formula, we obtain

$$
h^{0}\left(K_{S}+v\right) \geq \frac{\left(K_{S}+v\right) \cdot v}{2}+\chi\left(\mathcal{O}_{S}\right)=1
$$


If $K_{S}+v \sim_{\mathbb{Q}} 0$ in $\operatorname{Pic}(S)_{\mathbb{Q}}$, then $(3)$ clearly holds. If $K_{S}+v \nsim 0$ in $\operatorname{Pic}(S)_{\mathbb{Q}}$, then one can take the Zariski decomposition

$$
K_{S}+v=P+N
$$

in $\Lambda_{\mathbb{Q}}$ (see eg. [1, Theorem] whose proof is valid over any field $k$ ). Now the same proof of [26, Lemma 4.3, Page 175] implies the result. Indeed, $g^{*}(P)=P$ in $\Lambda_{\mathbb{Q}}$ and $g^{*} N=N$ by $g^{*}\left(K_{S}+v\right)=K_{S}+v$ and by the uniqueness of the Zariski decomposition. Then, by $(1), P=a v$ in $\Lambda_{\mathbb{Q}}$ for some rational number $a$. The rational number $1-a \in \mathbb{Q}$ is positive, since a smooth rational surface $S$ has $K_{S}$ non-pseudo effective. Replacing $v$ by $(1-a) v$ and putting $N(S):=N$, we obtain (3). Precisely, we have $g^{*} N=N$ where $N$ is regarded as in $\Lambda_{\mathbb{Q}} \subset \mathrm{NS}\left(X_{\bar{\eta}}\right)_{\mathbb{Q}}=\operatorname{Pic}\left(X_{\bar{\eta}}\right)_{\mathbb{Q}}$ (with the last equality due to $S$ being geometrically rational). Since $\left(N_{i} \cdot N_{j}\right)$ is negative definite (by the definition of the Zariski decomposition), we have $g^{*} N=N$ as Weil $\mathbb{Q}$-divisors.

Proposition 5.2. The case $q(X)=1$ in Proposition 3.2 does not occur.

Proof. Suppose the contrary that the case $q(X)=1$ in Proposition 3.2 occurs, we shall derive a contradiction.

For the $\sigma$-equivariant fibration $f: X \rightarrow Y$ there, let $X_{y}$ be a fibre over $y \in Y$. Then $F=X_{y}$ is a smooth rational surface and $Y$ is an elliptic curve. For the wild automorphism action of $\sigma$ on the elliptic curve $Y$, we have that $\sigma$ is a translation of infinite order.

Let $\eta \in Y$ be the scheme generic point of $Y$ and $X_{\eta}=f^{-1}(\eta)$ the scheme generic fibre of $f$. Since $\left.\mathrm{NS}(X)\right|_{X_{\eta}}=\mathrm{NS}\left(X_{\eta}\right)$ (cf. Lemma 2.14), we consider the natural action $\sigma^{*}$ on

$$
\Lambda:=\left.\mathrm{NS}(X)\right|_{X_{\eta}}=\mathrm{NS}\left(X_{\eta}\right)
$$

defined as:

$$
\sigma^{*}\left(\left.M\right|_{X_{\eta}}\right):=\left.\left(\sigma^{*} M\right)\right|_{X_{\eta}} .
$$

We can also define a pairing on $\Lambda$ by

$$
\left(\left.\left.M_{1}\right|_{X_{\eta}} \cdot M_{2}\right|_{X_{\eta}}\right)_{X_{\eta}} \cdot
$$

This paring is compatible with that on $\operatorname{NS}\left(X_{\eta}\right)$. It is non-degenerate and has signature $(1, r-1)$ with $r=\operatorname{rank} \Lambda=\operatorname{rank} \mathrm{NS}\left(X_{\eta}\right)$.

By the flatness of $f$, we have

$$
\left(\left.\left.M_{1}\right|_{X_{\eta}} \cdot M_{2}\right|_{X_{\eta}}\right)_{X_{\eta}}=\left(\left.\left.M_{1}\right|_{F} \cdot M_{2}\right|_{F}\right)_{F}=\left(M_{1} \cdot M_{2} \cdot F\right)_{X} .
$$

Here $F$ is any closed fibre of $f$.

Since $\sigma^{*} F \equiv F$ (numerical equivalence), we have

$$
\left(\sigma^{*}\left(\left.M_{1}\right|_{X_{\eta}}\right) \cdot \sigma^{*}\left(\left.M_{2}\right|_{X_{\eta}}\right)\right)=\left(\sigma^{*} M_{1} \cdot \sigma^{*} M_{2} \cdot F\right)_{X}=\left(M_{1} \cdot M_{2} \cdot F\right)_{X}=\left(\left.\left.M_{1}\right|_{X_{\eta}} \cdot M_{2}\right|_{X_{\eta}}\right) .
$$


Hence the action of $\sigma$ on $\Lambda$ is an isometry with respect to its pairing and the spectral radius $\rho\left(\left.\sigma^{*}\right|_{\Lambda}\right)$ is just the first relative dynamical degree $d_{1}\left(\left.\sigma\right|_{f}\right)$ defined in [4]. In particular, we have

$$
d_{1}(\sigma)=\rho\left(\left.\sigma^{*}\right|_{\Lambda}\right)
$$

by the product formula, because $Y$ is a curve with $d_{1}\left(\left.\sigma\right|_{Y}\right)=1$.

Run the relative minimal model program (MMP) over the elliptic curve $Y$. Let $X \rightarrow X_{1}$ be the contraction of a $K_{X}$-negative extremal ray over $Y$. Then $\rho(X)=\rho\left(X_{1}\right)+1$.

Suppose that the contraction $X \rightarrow X_{1}$ over $Y$ is a Fano contraction, i.e., a Mori fibre space. Note that every variety has only finitely many Fano contractions of extremal rays (cf. [25, Theorem 2.2]). Replacing $\sigma$ by a positive power, we may assume that $\sigma$ stabilizes the extremal ray of the contraction $X \rightarrow X_{1}$ and hence the action of $\sigma$ descends to that on $X_{1}$ (cf. e.g. [27, Lemma 2.12]) which is again wild, see Lemma 2.5.

If $\operatorname{dim} X_{1} \leq 1$, then $X_{1}=Y$, the fibration $X \rightarrow Y$ is a Fano contraction and $\rho(X)=2$. Now $\mathrm{NS}(X)_{\mathbb{Q}}$ is spanned by $-K_{X}$ and a fibre $X_{y}$ over $y \in Y$ both classes of which are $\sigma$-stable. So $\left.\sigma^{*}\right|_{\mathrm{NS}(X)}$ is of finite order. Thus $X$ is an abelian variety by Corollary 2.4 , contradicting the existence of a Fano contraction $X \rightarrow Y$.

If $\operatorname{dim} X_{1}=2$, then $X_{1}$ is an abelian surface by Corollary 3.1. However, then $1=$ $q(X) \geq q\left(X_{1}\right)=2$, a contradiction.

Thus we may assume that $X \rightarrow X_{1}$ is birational. Since a smooth threefold has no flip, $X \rightarrow X_{1}$ is a divisorial contraction with the exceptional locus $D_{0}$, a prime divisor, see [16]. Since we are running the relative MMP of $X$ over the elliptic curve $Y$, the intersection of $D_{0}$ with each fibre of $X \rightarrow Y$ is a union of several (rational) curves to be contracted by the map $X \rightarrow X_{1}$.

If the exceptional divisor $D_{0}$ is $\sigma$-periodic, the union of $\sigma^{i}\left(D_{0}\right)(i \geq 0)$ is a $\sigma$-stable Zariski-closed proper subset of $X$, a contradiction. Therefore, $D_{0}$ is not $\sigma$-periodic.

Now the proposition follows from the three claims below.

Claim 5.3. $d_{1}\left(\left.\sigma\right|_{X}\right)=1$.

Proof. Suppose the contrary that $d_{1}\left(\left.\sigma\right|_{X}\right)>1$. Then, since the locus $D_{0}$ of the divisorial extremal contraction $X \rightarrow X_{1}$ is not $\sigma$-periodic, [13, Lemma 6.3, Theorem 1.7] proves the lifting of $\sigma$ to some $X^{\prime}$ with a $\sigma$-equivariant birational morphism $\rho_{\xi}: X^{\prime} \rightarrow X$, and the descending of $\sigma$ to some surface $S$ with a $\sigma$-equivariant surjective morphism $\tau: X^{\prime} \rightarrow S$. Both the action of $\sigma$ on $X^{\prime}$ and $S$ are wild; see Lemma 2.5. Now $S$ is an abelian surface by Corollary 3.1. However, then $1=q(X)=q\left(X^{\prime}\right) \geq q(S)=2$, a contradiction. Thus the claim is true.

From now on, we may assume that $\rho\left(\left.\sigma^{*}\right|_{\Lambda}\right)=d_{1}\left(\left.\sigma\right|_{X}\right)=1$. 
Claim 5.4. The action of $\sigma$ on the hyperbolic lattice $\Lambda$ is of infinite order.

Proof. Suppose the contrary that $\left.\sigma^{*}\right|_{\Lambda}$ is of finite order. Replacing $\sigma$ by a positive power, we may assume that $\sigma^{*}$ acts on $\Lambda$ as the identity. Note that $\sigma^{-1} D_{0}$ is the exceptional locus of another divisorial contraction of extremal ray on $X$, see e.g. [27, Lemma 2.11]. Let

$$
X_{\bar{\eta}}=X_{\eta} \times_{\operatorname{Spec} \kappa(\eta)} \operatorname{Spec} \overline{\kappa(\eta)}
$$

be the geometric generic fibre of $f$. Since both $\left.D_{0}\right|_{X_{\bar{\eta}}}$ and $\left.\left(\sigma^{-1} D_{0}\right)\right|_{X_{\bar{\eta}}}$ are defined over $X_{\eta}$, it follows from $\sigma^{*}=$ id on $\Lambda\left(\subset \operatorname{NS}\left(X_{\bar{\eta}}\right)\right)$ that

$$
\left.\left(D_{0}\right)\right|_{X_{\bar{\eta}}}=\left.\left(\sigma^{*} D_{0}\right)\right|_{X_{\bar{\eta}}} \in \mathrm{NS}\left(X_{\bar{\eta}}\right)
$$

On the other hand, both $\left.\left(D_{0}\right)\right|_{X_{\bar{\eta}}}$ and $\left.\left(\sigma^{*} D_{0}\right)\right|_{X_{\bar{\eta}}}$ are unions of a few contractible curves on the smooth rational projective surface $X_{\bar{\eta}}$. The negativity of $D_{0 \mid X_{\bar{\eta}}}$ implies that $\left.D_{0}\right|_{X_{\bar{\eta}}}=\left.\left(\sigma^{-1}\left(D_{0}\right)\right)\right|_{X_{\bar{\eta}}}$ as sets. Then $\left.D_{0}\right|_{X_{\eta}}=\left.\left(\sigma^{-1}\left(D_{0}\right)\right)\right|_{X_{\eta}}$ as divisors on $X_{\eta}$. By taking the Zariski closure, we obtain $D_{0}=\sigma^{-1}\left(D_{0}\right)$, as both $D_{0}$ and $\sigma^{-1} D_{0}$ are irreducible divisors on $X$. However, this contradicts the fact that $D_{0}$ is not $\sigma$-periodic ( $\sigma$ being wild). This proves the claim.

To finish the proof of Proposition 5.2, we still have to prove the following.

Claim 5.5. It is impossible that the action of $\sigma$ on the hyperbolic lattice $\Lambda$ is parabolic, i.e., it is of infinite order and $\rho\left(\left.\sigma^{*}\right|_{\Lambda}\right)=d_{1}\left(\left.\sigma\right|_{X}\right)=1$.

Proof. Suppose the contrary that the action of $\sigma$ on $\Lambda$ is parabolic. We apply Lemma 5.1 to Case $q(X)=1$ in Proposition 3.2 with $S=X_{\eta}$ the generic fibre of $f: X \rightarrow$ $Y=\operatorname{Alb}(X)$, and $g^{*}$ equal to $\sigma^{*}: \mathrm{NS}(X) \rightarrow \mathrm{NS}(X)$ restricted to the subspace $\Lambda=$ $\left.\operatorname{NS}(X)\right|_{X_{\eta}}=\operatorname{NS}\left(X_{\eta}\right)$ of the space $\operatorname{NS}\left(X_{\bar{\eta}}\right)=\operatorname{Pic}\left(X_{\bar{\eta}}\right)$. Then, by Lemma 5.1 , there is a nonzero nef $\mathbb{Q}$-divisor $v=\left.V\right|_{X_{\eta}}$ in $\Lambda$ such that $v^{2}=0,\left.\left(K_{X}+V\right)\right|_{X_{\eta}} \sim_{\mathbb{Q}} N\left(X_{\eta}\right)$, and $\sigma^{*}\left(N\left(X_{\eta}\right)\right)=N\left(X_{\eta}\right)$. It follows that the Zariski closure of the image of $N\left(X_{\eta}\right)$ in $X$ is a $\sigma$-stable proper closed subset of $X$. Thus $N\left(X_{\eta}\right)=0$ since the wild automorphism $\sigma$ has no non-empty closed proper subset of $X$. Hence $\left.\left(K_{X}+V\right)\right|_{X_{\eta}} \sim_{\mathbb{Q}} 0$. Now $-\left.K_{X_{\eta}} \sim V\right|_{X_{\eta}}$ is nef with zero self-intersection. Hence $\kappa\left(X_{\eta},-K_{X_{\eta}}\right) \in\{0,1\}$ by the Riemann-Roch theorem.

If $\kappa\left(X_{\eta},-K_{X_{\eta}}\right)=0$, then $\sigma$ stabilises the unique member in $\left|-K_{X_{\eta}}\right|$ and its closure on $X$, contradicting that $\sigma$ is wild. If $\kappa\left(X_{\eta},-K_{X_{\eta}}\right)=1$, then a multiple of the nef $-K_{X_{\eta}}$ supports fibres of the unique (relatively minimal) elliptic fibration on $X_{\eta}$. This fibration has singular fibres by Kodaira's canonical bundle formula for elliptic (rational) surfaces. Now $\sigma$ stabilises the union (and its closure in $X$ ) of singular fibres. This contradicts that $\sigma$ is wild. The claim is proved. 
This proves Proposition 5.2.

\section{Proof of Theorem 1.5}

Lemma 6.1. Let $X$ be a Calabi-Yau manifold of dimension three. Suppose $\sigma$ is a wild automorphism of $X$. Then $K_{X}$ is trivial in $\operatorname{Pic}(X)$ and $\sigma$ has zero entropy.

Proof. Since $\chi\left(\mathcal{O}_{X}\right)=0$ by Proposition 2.6 and $h^{1}\left(\mathcal{O}_{X}\right)=0$ by assumption, it follows that $h^{3}\left(\mathcal{O}_{X}\right)>0$. Hence $h^{0}\left(\mathcal{O}_{X}\left(K_{X}\right)\right)=h^{0}\left(\mathcal{O}_{X}\right)>0$ by the Serre duality. Thus $K_{X}=0$ in $\operatorname{Pic}(X)$ as $K_{X}$ is $\mathbb{Q}$-linearly equivalent to 0 .

We show that $\sigma$ has zero entropy. Indeed, by [4], replacing $X$ by the universal cover and $\sigma$ by its lifting, we may assume that $X$ is a Calabi-Yau threefold in the strict sense. Since $h^{1}\left(\mathcal{O}_{X}\right)=0$, we have $b_{5}(X)=b_{1}(X)=0$. Thus, under the notation of Propoition 2.16, we have $A=H^{3}(X, \mathbb{Z}) /($ torsion). By Proposition 2.16, it suffices to show that the complex eigenvalues of $\left.\sigma^{*}\right|_{A}$ are roots of the unity.

Again, by $b_{5}(X)=0$, the cohomology group $H^{3}(X, \mathbb{C})$ is primitive. So is each Hodge component $H^{p, q}(X)$ of $H^{3}(X, \mathbb{C})$. Thus the product on $H^{2,1}(X)$ defined below

$$
H^{2,1}(X) \times H^{2,1}(X) \ni(\eta, \eta) \mapsto \eta \wedge \bar{\eta}
$$

is, up to the following unit (with $(p, q ; n, k)=(2,1 ; 3,0))$

$$
c_{p, q ; n, k}:=(\sqrt{-1})^{p-q}(-1)^{(n-k)(n-k-1) / 2},
$$

a definite Hermitian form by the Hodge-Riemann relation as in [8, page 123]. Hence the action of $\sigma^{*}$ on $H^{1,2}(X) \oplus H^{2,1}(X)=H^{1,2}(X) \oplus \overline{H^{1,2}(X)}$ is unitary. For the same reason, the action of $\sigma^{*}$ on $H^{3,0}(X) \oplus H^{0,3}(X)=H^{3,0}(X) \oplus \overline{H^{3,0}(X)}$ is also unitary. So the eigenvalues of the action of $\sigma$ on $H^{3}(X, \mathbb{C})$ are of absolute value 1 . Therefore, they are roots of the unity since the action of $\sigma^{*}$ is defined on the integral $H^{3}(X, \mathbb{Z})$ and by Kronecker's theorem. This proves the lemma.

Lemma 6.2. Let $X$ be a projective variety of dimension $\leq 4$ such that the Kodaira dimension $\kappa(X) \geq 0$. Suppose $\sigma$ is a wild automorphism of $X$. Then $\sigma$ has zero entropy.

Proof. By Proposition 2.6, $K_{X} \sim_{\mathbb{Q}} 0$ and we may assume that $X$ coincides with its Beauville-Bogomolov minimal split cover and that each factor $X_{i}$ of $X$ is stable under $\sigma$ so that $\left.\sigma\right|_{X_{i}}$ is wild. Then $\chi\left(\mathcal{O}_{X_{i}}\right)=0$ by Proposition 2.6 (1) and also $\operatorname{dim} X_{i} \leq 4$. Therefore $X_{i}$ is either an abelian variety or a Calabi-Yau threefold in the strict sense (Proposition 2.6). Note that $\sigma$ has zero entropy if and only if $\left.\sigma\right|_{X_{i}}$ has zero entropy for every factor $X_{i}$. If $X_{i}$ is a Calabi-Yau threefold, then $\left.\sigma\right|_{X_{i}}$ has zero entropy by Lemma 6.1. If $X_{i}$ is an abelian variety, then $\left.\sigma\right|_{X_{i}}$ has zero entropy by [21, Theorem 0.2]. This proves the lemma. 
Lemma 6.3. Let $X$ be a projective variety over $\mathbb{C}$ of dimension four such that $q(X)=0$. Suppose $\sigma$ is a wild automorphism of $X$. Then $\sigma$ has zero entropy.

Proof. By Lemma 2.5, $X$ is smooth. Let $H^{i}(X, \mathbb{Z})_{f}:=H^{i}(X, \mathbb{Z}) /($ torsion $)$ be the free part of $H^{i}(X, \mathbb{Z})$. We need to show the claim that $d_{1}(\sigma)=1$.

Note that $\sigma^{*}$ preserves the closure of the Kähler cone of $X$ and the Kähler cone spans $H^{1,1}(X, \mathbb{R})$. Thus, by the Perron-Frobenius theorem, $\sigma^{*} L_{\sigma}=d_{1}(\sigma) L_{\sigma}$ for some nonzero class $L_{\sigma}$ in the closure of the Kähler cone of $X$, called a nef class. Of course, $L_{\sigma} \in$ $H^{1,1}(X, \mathbb{R}) \subset H^{2}(X, \mathbb{R})$.

Here and hereafter, we note that $\sigma$ preserves $H^{i, j}(X, \mathbb{C})$ in the Hodge decomposition

$$
H^{r}(X, \mathbb{C})=\oplus_{i+j=r} H^{i, j}(X, \mathbb{C})
$$

and we denote by

$$
H^{r}(X, K)=H^{r}(X, \mathbb{Z}) \otimes_{\mathbb{Z}} K
$$

for $K=\mathbb{Q}, \mathbb{R}, \mathbb{C}$.

Let $a_{i}(1 \leq i \leq r)$ be the complex eigenvalues of $\left.\sigma^{*}\right|_{H^{2}(X, \mathbb{Z})_{f}}$ counted with multiplicities. Then one of these $a_{i}$ is $d_{1}(\sigma)$; this is because these $a_{i}$ are also the eigenvalues of $H^{2}(X, K)$ for $K=\mathbb{Q}, \mathbb{R}$ or $\mathbb{C}$. Then the complex eigenvalues of $\left.\left(\sigma^{-1}\right)^{*}\right|_{H^{2}(X, \mathbb{Z})_{f}}$ are $a_{i}^{-1}(1 \leq i \leq r)$ counted with multiplicities. Since $H^{2}(X, \mathbb{Z})_{f}$ and $H^{6}(X, \mathbb{Z})_{f}$ are dual under the cup product, the complex eigenvalues of $\left.\sigma^{*}\right|_{H^{6}(X, \mathbb{Z})_{f}}$ are also $a_{i}^{-1}(1 \leq i \leq r)$ counted with multiplicities. Then,

$$
1 \leq d_{1}(\sigma)=a_{i}, 1 \leq d_{3}(\sigma)=d_{1}\left(\sigma^{-1}\right)=a_{j}^{-1}
$$

for some $1 \leq i, j \leq r$ possibly $i=j$. If $i=j$, then $d_{1}(\sigma)=1$. This is because $a_{i}=1$ derived from $1 \leq a_{i}$ and $1 \leq a_{i}^{-1}$ if $i=j$.

So, from now on we may assume that $i \neq j$. Then, by renumbering $a_{i}(1 \leq i \leq r)$ and by interchanging $\sigma$ and $\sigma^{-1}$ if necessary, we may assume that

$$
d_{1}(\sigma)=a_{1} \geq a_{r}^{-1}=d_{1}\left(\sigma^{-1}\right)=d_{3}(\sigma) \geq 1
$$

Recall that $b_{7}(X)=b_{1}(X)=0$ by the assumption. Then, by Proposition 2.16, $a_{1}$ is also an eigenvalue of either $\left.\sigma^{*}\right|_{H^{3}(X, \mathbb{Z})_{f}}$ or $\left.\sigma^{*}\right|_{H^{5}(X, \mathbb{Z})_{f}}$.

Assume first that $a_{1}$ is an eigenvalue of $\left.\sigma^{*}\right|_{H^{3}(X, \mathbb{Z})_{f}}$. Choose an eigenvector $0 \neq u \in$ $H^{3}(X, \mathbb{C})$. Since $\sigma^{*}$ preserves the Hodge decomposition, we may choose $u$ so that $u \in$ $H^{p, q}(X)$ in some Hodge component $H^{p, q}(X)$ of $H^{3}(X, \mathbb{C})$. Consider the complex conjugate $\bar{u}$ of $u$ with respect to $H^{3}(X, \mathbb{R}) \otimes_{\mathbb{R}} \mathbb{C}=H^{3}(X, \mathbb{C})$. Then $\bar{u} \in H^{q, p}(X)$ and $\sigma^{*} \bar{u}=a_{1} \bar{u}$, as $\sigma^{*}$ is defined over $H^{3}(X, \mathbb{R})$ and $a_{1}$ is real. 
On the other hand, $H^{3}(X, \mathbb{Z})$ is primitive by $b_{7}(X)=b_{1}(X)=0$. Hence the Hodge component $H^{p, q}(X)$ is also primitive. Then, as in the proof of Lemma 6.1, we have the unit $c_{p, q, n, k}$ as given there but with $(p, q ; n, k)=(p, q ; 4,1)$ and $p+q=3$ now, such that

$$
c_{p, q ; n, k}(v \cdot \bar{v} \cdot \eta)>0
$$

for every $v \in H^{p, q}(X) \backslash\{0\}$ and for every Kähler class $\eta \in H^{1,1}(X, \mathbb{R})$ by the HodgeRiemann relation for the primitive cohomology group $H^{p, q}(X)([8$, page 123$])$. In particular,

$$
u \bar{u} \neq 0
$$

in $H^{3,3}(X, \mathbb{R})$ and satisfies

$$
\sigma^{*}(u \bar{u})=a_{1}^{2} u \bar{u} .
$$

Thus, $a_{1}^{2}$ is a positive real eigenvalue of $\left.\sigma^{*}\right|_{H^{3,3}(X, \mathbb{R})}$. Since $d_{3}(\sigma)=a_{r}^{-1}$ is the spectral radius of $\left.\sigma\right|_{H^{6}(X, \mathbb{R})}$, it follows that

$$
a_{1}^{2} \leq d_{3}(g)=a_{r}^{-1} \leq a_{1} .
$$

For the last inequality, we used our assumption that $a_{1} \geq a_{r}^{-1} \geq 1$. Hence $a_{1}=1$ again, since $1 \leq a_{1}$. Thus $d_{1}(\sigma)=1$ as claimed.

Next we consider the case where $a_{1}$ is an eigenvalue of $\left.\sigma^{*}\right|_{H^{5}(X, \mathbb{Z})_{f}}$. Then $a_{1}$ is an eigenvalue of $\left.\left(\sigma^{-1}\right)^{*}\right|_{H^{3}(X, \mathbb{Z})_{f}}$ by the duality. Then applying the same argument as above for $\sigma^{-1}$, we find that there are a Hodge component $H^{p, q}(X)$ of $H^{3}(X, \mathbb{C})$ and a non-zero element $u \in H^{p, q}(X)$ such that $u \bar{u} \in H^{3,3}(X, \mathbb{R})$ is an eigenvector of $\left.\left(\sigma^{-1}\right)^{*}\right|_{H^{3,3}(X, \mathbb{R})}$ corresponding to an eigenvalue $a_{1}^{2}$, which is real and positive. Thus,

$$
a_{1}^{2} \leq d_{3}\left(\sigma^{-1}\right)=d_{1}(\sigma)=a_{1} .
$$

Since $1 \leq a_{1}$, it follows that $a_{1}=1$. Hence $d_{1}(\sigma)=1$ also in this case, as claimed.

This proves the lemma.

Proposition 6.4. Let $X$ be a projective variety over $\mathbb{C}$ with a wild automorphism $\sigma$.

(1) Suppose $\operatorname{dim} X \leq 3$. Then $X$ is either an abelian variety or a Calabi-Yau threefold with the canonical divisor $K_{X} \sim 0$. Moreover $\sigma$ has zero entropy.

(2) Suppose $\operatorname{dim} X=4$. Then $\sigma$ has zero entropy, unless the Kodaira dimension $\kappa(X)=-\infty$ and the irregularity $q(X)>0$.

Proof. By Theorem 3.1 and Propositions 3.2, 4.1 and 5.2, we already know that a projective variety $X$ with $\operatorname{dim} X \leq 3$ and with a wild automorphism is isomorphic to either an abelian variety or a Calabi-Yau threefold. Thus Proposition 6.4 (1) follows from Lemma 6.1. Proposition 6.4 (2) follows from Lemmas 6.2 and 6.3 .

6.5. Proof of Theorem 1.5. It follows from Proposition 6.4 and Proposition 2.15. 


\section{Wild AUTOMORPHISMS OF ZERO ENTROPY}

In this section, we consider the case where $X$ is a (smooth) projective variety with a wild automorphism $\sigma$ such that $\sigma$ is of zero entropy.

Lemma 7.1. Let $\sigma$ be an automorphism of zero entropy of a smooth projective variety. Then the following three conditions are equivalent.

(1) $\sigma$ is wild.

(2) The twisted homogeneous coordinate ring $B(X, L, \sigma)$ is projectively simple for any $\sigma$-ample line bundle $L$ on $X$.

(3) The twisted homogeneous coordinate ring $B(X, L, \sigma)$ is projectively simple for at least one ample line bundle $L$ on $X$.

Proof. By [9, Theorem 1.2 (2)], any ample line bundle on $X$ is $\sigma$-ample, because $\sigma$ is of zero entropy. Thus, the implication $(2) \Rightarrow(3)$ is clear and the implications $(1) \Rightarrow(2)$ and $(3) \Rightarrow(1)$ follow from [21, Proposition 0.1].

Lemma 7.2. Let $X$ be a (smooth) projective variety. Suppose that $\sigma$ is a wild automorphism of $X$ of zero entropy. Then $\left.\sigma^{*}\right|_{\mathrm{NS}(X)_{\mathbb{C}}}$ is not diagonalizable, unless $X$ is isomorphic to an abelian variety.

Proof. We embed the lattice $\Lambda:=\mathrm{NS}(X) /$ (torsion) in $H^{1,1}(X \mathbb{R}) \subset H^{1,1}(X, \mathbb{C})$. By the assumption, $d_{1}(\sigma)=1$, so the spectral radius of the pullback action $\sigma^{*}$ on $H^{1,1}(X, \mathbb{C})$ and hence that on $\Lambda$ are 1. Thus every eigenvalue of $\left.\sigma^{*}\right|_{\Lambda}$ is an algebraic integer of modulus 1. Hence, by Kronecker's theorem, all eigenvalues of $\Lambda$, or equivalently of $\left.\sigma^{*}\right|_{\mathrm{NS}(X)_{\mathbb{C}}}$, are roots of unity.

So, if $\left.\sigma^{*}\right|_{\mathrm{NS}(X)_{\mathbb{C}}}$ is diagonalizable, then $\left(\sigma^{*}\right)^{m}=$ id on $\mathrm{NS}(X)$ for some $m>0$. In this case, $X$ is an abelian variety by Corollary 2.4. This proves the lemma.

The following conjecture is in [18, Question 2.6].

Conjecture 7.3. (cf. [18]) Every nef $\mathbb{Q}$-Cartier divisor L (not necessarily effective) on a Calabi-Yau manifold of dimension three is $\mathbb{Q}$-linearly equivalent to an effective divisor (and hence semi-ample by the known log Abundance in dimension $\leq 3$ ).

The following is asserted in [10].

Theorem 7.4. (cf. [10, Theorem 4.7], and also Remark 7.5) Assume Conjecture 7.3 for 3-dimensional Calabi-Yau manifolds in the strict sense. Let $X$ be a (smooth) Calabi-Yau threefold. Then $X$ does not admit a wild automorphism.

Remark 7.5. About the proof of [10, Theorem 4.7], and the necessity of $\mathbb{Q}$-Cartier assumption on $L$ in Conjecture 7.3, we have: 
(1) In the proof of [10, Theorem 4.7], the author proceeds by asserting the existence of a nef divisor $L$ with $c_{2}(X) \cdot L=0$ and claims that such $L$ is semi-ample by applying Conjecture 7.3. This argument does not seem to work because such $L$ may not be a $\mathbb{Q}$-divisor (as required) to apply the conjecture.

(2) Indeed, Conjecture 7.3 does not hold for $\mathbb{R}$-Cartier divisors. Precisely, in [20, Theorem 1.4, Proposition 4.4], the authors constructed an automorphism $f$ of positive entropy on a (smooth) Calabi-Yau 3-fold $X$ such that $f$ is primitive, i.e., there is no $f$-equivariant rational map to any variety $Y$ with $0<\operatorname{dim} Y<\operatorname{dim} X$. By Perron-Frobenius theorem, there is a nonzero nef $\mathbb{R}$-Cartier divisor $L$ such that $f^{*} L \equiv d_{1}(f) L$. Since $d_{1}(f)>1$, we have $c_{2}(X) . L=0$. But this $L$ is not semi-ample, or else it would have induced an $f$-equivariant non-trivial morphism to a curve or a surface, contradicting the primitivity of $f$.

(3) For the sake of completeness, in the next paragraphs, we supply a proof of [10, Theorem 4.7].

Lemma 7.6. Let $X$ be a normal projective variety and $\sigma$ an automorphism. Suppose $\left.\sigma^{*}\right|_{\mathrm{NS}(X)_{\mathbb{C}}}$ is of infinite order and $\sigma$ is of zero entropy. Then there is a nef (integral) Cartier divisor $L \not \equiv 0$ such that $\sigma^{*} L \equiv L$ (numerical equivalence) possibly after replacing $\sigma$ by a positive power. In particular, we may assume $\sigma^{*} L \sim L$ if the irregularity $q(X)=0$.

Proof. Replacing $\sigma$ by a positive power, we may assume that $\left.\sigma^{*}\right|_{\mathrm{NS}(X)_{\mathbb{C}}}$ is unipotent (but not trivial) so it is equal to $I+N$ with $N$ nilpotent. Let $m(\geq 2)$ be the maximal order of Jordan blocks of $\left.\sigma^{*}\right|_{\mathrm{NS}(X)_{\mathbb{C}}}$. Then $N^{m}=0$, but $N^{m-1} \neq 0$. Thus

$$
g:=\lim _{s \rightarrow \infty}\left(\sigma^{*}\right)^{s} / s^{m-1}=N^{m-1} /(m-1) !
$$

is a nonzero linear transformation on $\mathrm{NS}(X)_{\mathbb{Q}}$. Since ample Cartier divisor classes generate $\mathrm{NS}(X)_{\mathbb{Q}}$ and since $g=N^{m-1} /(m-1) ! \neq 0$ on $\mathrm{NS}(X)_{\mathbb{Q}}$, there is an ample Cartier divisor $H$ on $X$ so that $L:=g(H)$ is nonzero $\mathbb{Q}$-Cartier divisor. Since $L_{s}:=\left(\sigma^{*}\right)^{s}(H) / s^{m-1}$ is ample, $L=\lim _{s \rightarrow \infty} L_{s}$ is nef. Moreover, using $\sigma^{*}=I+N$ and $N^{m}=0$, we compute

$$
\sigma^{*} L=\lim _{s \rightarrow \infty}(I+N)^{s+1}(H) / s^{m-1}=\left(N^{m-1} /(m-1) !\right)(H)=g(H)=L .
$$

The lemma follows by replacing $L$ by a positive multiple.

7.7. Proof of Theorem 7.4. Let $X$ be a (smooth) Calabi-Yau threefold with a wild automorphism $\sigma$. By Proposition 6.4, $\sigma$ is of zero entropy. We are going to reach a contradiction. By Proposition 2.6, we can lift $\sigma$ to $\widetilde{\sigma}$ on the universal cover $\widetilde{X}$ of $X$, such that $\tilde{X}$ is a Calabi-Yau manifold in the strict sense (while $\widetilde{\sigma}$ is, of course, still of zero entropy). Replacing $X$ by $\widetilde{X}$ we may assume that $X$ is a Calabu-Yau manifold in the strict sense. 
By Lemma 7.6, we have an integral nef Cartier divisor $L \not \equiv 0$ such that $\sigma^{*} L \sim L$. Since we have assumed that Conjecture 7.3 holds for such $X$, we have $|m L| \neq \emptyset$ for some positive integer $m$, contradicting Proposition 2.6 (2). This proves Theorem 7.4.

\section{REFERENCES}

[1] T. Bauer, A simple proof for the existence of Zariski decompositions on surfaces, J. Algebraic Geom. 18 (2009), no. 4, 789 - 793. 16

[2] A. Beauville, Some remarks on Kähler manifolds with $c_{1}=0$, Classification of Algebraic and Analytic Manifolds (Katata, 1982, ed. K. Ueno), Progr. Math., 39 Birkhäuser 1983, pp. 1 - 26. 6, 8

[3] F. Campana, J. A. Chen and T. Peternell, Strictly nef divisors, Math. Ann. 342 (2008), no. 3, 565 $-585.8$

[4] T. -C. Dinh, V. -A. Nguyen, and T. Truong, On the dynamical degree of meromorphic maps preserving a fibration, Commun. Contemp. Math. 14 (2012), no. 6, 18 pp. 10, 17, 19

[5] T. C. Dinh, F. Hu and D. -Q. Zhang, Compact Kähler manifolds admitting large solvable groups of automorphisms, Adv. Math. 281 (2015) 333 - 352. 4

[6] A. Fujiki, On automorphism groups of compact Kähler manifolds, Invent. Math. 44 (1978), no. 3, $225-258.4$

[7] T. Graber, J. Harris and J. Starr, Families of rationally connected varieties, J. Amer. Math. Soc. 16 (2003), no. $1,57-67.8$

[8] P. Griffiths and J. Harris, Principles of algebraic geometry, Pure and Applied Mathematics, WileyInterscience (John Wiley \& Sons), New York, 1978. 19, 21

[9] D. Keeler, Criteria for $\sigma$-ampleness, J. Amer. Math. Soc. 13 (2000), no. 3, 517 - 532. 2, 3, 22

[10] A. Kirson, Wild automorphisms of varieties with Kodaira dimension 0, Ann. Univ. Ferrara Sez. VII Sci. Mat. 56 (2010), no. 2, 327 - 333. 2, 8, 22, 23

[11] J. Kollár, Rational curves on algebraic varieties, Ergebnisse der Mathematik und ihrer Grenzgebiete. 3. Folge. Vol. 32, Springer-Verlag, Berlin, 1996. 7

[12] J. Kollár and S. Mori, Birational geometry of algebraic varieties, Cambridge Tracts in Math. 134, Cambridge Univ. Press, 1998. 7

[13] J. Lesieutre, Some constraints of positive entropy automorphisms of smooth threefolds, Ann. Sci. Éc. Norm. Supér. (4) 51 (2018), no. 6, 1507 - 1547. 17

[14] S. Li, Derived length of zero entropy groups acting on projective varieties in arbitrary characteristic - a remark to a paper of Dinh-Oguiso-Zhang, Internat. J. Math. 31 (2020), no. 8, 2050059, 10 pp. 4

[15] D. I. Lieberman, Compactness of the Chow scheme: applications to automorphisms and deformations of Kähler manifolds, Fonctions de plusieurs variables complexes, III (Sém. François Norguet, 1975-1977), Lecture Notes in Math., vol. 670, Springer, Berlin, 1978, pp. 140-186. 4

[16] S. Mori, Threefolds whose canonical bundles are not numerically effective, Ann. of Math. (2) 116 (1982), no. 1, 133-176. 17

[17] N. Nakayama, Intersection sheaves over normal schemes, J. Math. Soc. Japan 62 (2010), no. 2, 487 - 595. 3,7

[18] K. Oguiso, On algebraic fiber space structures on a Calabi-Yau 3-fold, Internat. J. Math. 4 (1993) 439 - 465. 2,22 
[19] K. Oguiso, Automorphisms of hyperkähler manifolds in the view of topological entropy, Algebraic geometry, Contemp. Math., vol. 422, Amer. Math. Soc., Providence, RI, 2007, pp. 173 - 185. 15

[20] K. Oguiso and T. T. Truong, Explicit examples of rational and Calabi-Yau threefolds with primitive automorphisms of positive entropy, J. Math. Sci. Univ. Tokyo 22 (2015), no. 1, 361 - 385. 23

[21] Z. Reichstein, D. Rogalski and J. J. Zhang, Projectively simple rings, Adv. Math. 203 (2006), no. 2, 365 - 407. 1, 2, 3, 4, 5, 6, 10, 13, 14, 15, 19, 22

[22] F. Sakai, The structure of normal surfaces, Duke Math. J. 52 (1985), no. 3, 627 - 648. 11, 13

[23] T. Suwa, On ruled surfaces of genus 1, J. Math. Soc. Japan 21 (1969) 291 - 311.12

[24] K. Ueno, Classification theory of algebraic varieties and compact complex spaces, Lecture Notes in Mathematics, Vol. 439, Springer-Verlag, Berlin-New York, 1975. 6

[25] J. A. Wisniewski, On contractions of extremal rays of Fano manifolds, J. Reine Angew. Math. 417 (1991), 141-157. 17

[26] D. -Q. Zhang, Automorphism groups and anti-pluricanonical curves, Math. Res. Lett. 15 (2008), no. $1,163-183.16$

[27] D. -Q. Zhang, Polarized endomorphisms of uniruled varieties, With an appendix by Y. Fujimoto and N. Nakayama, Compos. Math. 146 (2010), no. 1, 145 - 168. 17, 18

Department of Mathematical Sciences, the University of Tokyo, Meguro Komaba 3-81, Tokyo, Japan, and National Center for Theoretical Sciences, Mathematics Division, National Taiwan University, Taipei, Taiwan

Email address: oguiso@g.ecc.u-tokyo.ac.jp

Department of Mathematics, National University of Singapore, 10 Lower Kent Ridge Road, Singapore 119076, Republic of Singapore

Email address: matzdq@nus.edu.sg 